\title{
Norois
}

Environnement, aménagement, société

$217 \mid 2010 / 4$

Interroger les processus de valorisation des espaces urbains

\section{Déqualification, sécurisation et éviction au service de la réappropriation du centre historique de Gênes}

Disqualification, security production and eviction in favor of the reappropriation of the historical center of Genoa

\section{Sébastien Jacquot}

\section{(2) OpenEdition}

\section{Journals}

\section{Édition électronique}

URL : http://journals.openedition.org/norois/3451

DOI : $10.4000 /$ norois.3451

ISBN : 978-2-7535-1565-9

ISSN : 1760-8546

\section{Éditeur}

Presses universitaires de Rennes

\section{Édition imprimée}

Date de publication : 15 décembre 2010

Pagination : 29-39

ISBN : 978-2-7535-1340-2

ISSN : 0029-182X

\section{Référence électronique}

Sébastien Jacquot, « Déqualification, sécurisation et éviction au service de la réappropriation du centre historique de Gênes », Norois [En ligne], 217 | 2010/4, mis en ligne le 31 décembre 2012, consulté le 20 avril 2019. URL : http://journals.openedition.org/norois/3451 ; DOI : 10.4000/ norois.3451 


\title{
DÉQUALIFICATION, SÉCURISATION ET ÉVICTION
}

\section{AU SERVICE DE LA RÉAPPROPRIATION DU CENTRE HISTORIQUE DE GÊNES}

\author{
SÉBASTIEN JACQUOT \\ EA EIREST \\ (Université de Paris 1 Panthéon Sorbonne), \\ 12, place du Panthéon - 75005 PARIs, France \\ sebastien.jacquot@univ-paris1.fr
}

\section{RÉSUMÉ}

Le centre historique de Gênes connaît une phase de réappropriation via la patrimonialisation et le processus de gentrification d'une partie de ses espaces. Cette étude vise à montrer les dispositifs et techniques accompagnant et pérennisant cette mutation du statut des espaces, par leur sécurisation et l'éviction de certains usages et usagers. Toutefois ce processus intègre une dimension réflexive liée à la prégnance d'imaginaires locaux conférant une valeur positive à certaines formes de marginalités.

MOTS CLÉ : appropriation - déqualification - sécurisation - patrimonialisation - prostitution - imaginaires - requalification urbaine - Gênes

\section{ABSTRACT \\ Disqualification, security production and eviction in favor of the reappro- priation of the historical center of Genoa \\ The historic center of Genoa is undergoing a re-appropriation phenomenon through heritage politics and a gentrification process. This study aims to show the apparatus and techniques that are used to control and perpetuate the mutation of the status of spaces, by the multifocal production of urban security and the eviction of uses and users. However, this process incorporates a reflexive dimension related to local imaginaries giving a positive value to certain forms of marginality.}

KEY WORDS : appropriation - disqualification - security - heritage - prostitution - imaginaries - urban regeneration - Genoa

Après avoir connu une phase d'importante dévalorisation symbolique et sociale, les centres historiques anciens deviennent des espaces emblématiques des villes, notamment par l'action combinée de la patrimonialisation et du changement social (Tomas, 2004). La mise en tourisme et les mutations commerciales apparaissent comme signe et instrument d'une importante revalorisation symbolique et économique (Zukin, 1993), par le biais des changements d'usage. Ainsi, boutiques, hôtels de qualité et chambres d'hôte, restauration ou réhabilitation d'un bâti ancien, constituent autant de signes de ce réinvestissement des espaces historiques centraux. Ces transformations sont souvent envisagées du point de vue de leur terme qui semble inéluctable, à travers la diffusion d'une gentrification généralisée (Smith, 2002). Elles reposent sur le jeu noué entre les processus 
de revalorisation multidimensionnelle et l'éviction tendancielle des usages antérieurs, désormais considérés comme marginalités et voués à des localisations plus périphériques. Des processus de (dé)légitimation et déqualification symbolique accompagnent ces transformations sur le versant idéel. La notion d'« appropriation de l'espace » permet d'interpréter les mutations en cours en termes de dynamiques socio-spatiales (Ripoll et Veschambre, 2005).

Le caractère polysémique de la notion de valorisation indique en effet les enjeux complexes de ces mutations, car la valeur renvoie à la fois à l'évaluation économique (Greffe, 2003), notamment immobilière, et aux critères au nom desquels un ensemble urbain est considéré comme patrimoine (Riegl, 1984), permettant une mise en scène de l'appropriation comme redécouverte de valeurs cachées. En retour, cette mise en avant de valeurs plus immatérielles est un facteur essentiel de la revalorisation économique, notamment dans le cadre d'opérations de régénération urbaine (Vivant et Charmes, 2008).

L'étude des transformations du centre historique de Gênes ${ }^{1}$, objet de nombreuses interventions publiques et privées depuis les années 1980, permet de cerner les enjeux complexes de revalorisation et réappropriation, sur fond d'une déqualification des usages antérieurs, en montrant les processus et stratégies conduisant à une inversion de la valeur portée aux lieux, de la catégorisation de l'espace à la sécurisation et à l'éviction de pratiques et d'usagers considérés désormais comme illégitimes, notamment dans le cadre des tentatives de contrôle et suppression de la prostitution.

\section{La réappropriation spatiale sur fond de patrimonialisation}

La patrimonialisation des espaces combine des processus idéels et matériels, permettant de lier enjeux sociaux et de pouvoir au sein des villes. Ces transformations ne sont pas assignables à un acteur unique, combinant long terme et court terme, stratégies, dispositifs et imaginaires. La dévalorisation des espaces historiques est-elle alors un frein ou au contraire un préalable à une appropriation, en permettant la légitimation des transformations menées, par délégitimation et éviction des processus et appropriations antérieurs?

\section{LES MOTS DE LA DÉQUALIFICATION}

Le centre historique de Gênes existe comme entité depuis l'extension et la modernisation de la ville au $\mathrm{XIX}^{\mathrm{e}}$ siècle, qui font apparaître une ville ancienne et une ville moderne, mais ne naît comme catégorie urbanistique que dans le Piano Regolatore Generale de 1976. La déqualification préalable est facilitée par les multiples formes de dépréciation urbanistiques et sémantiques du centre historique.

Du milieu du XIX à la fin du XX ${ }^{\mathrm{e}}$ siècle se succèdent alors projets de destruction, d'assainissement ( risanamento»), de percées et d'éventrement ("sventramento »), justifiés par les épidémies de tuberculose au XIX $^{\mathrm{e}}$ siècle ou les nécessités de circulation de la vie moderne. L'hygiénisme et le modernisme demeurent longtemps les arguments en faveur des destructions de quartiers anciens : plusieurs quartiers médiévaux ont été rasés, laissant place à des constructions modernes. Ces opérations sont difficiles à mener : elles impliquent de larges expropriations et le relogement des familles, ce qui explique que certaines demeurent à l'état de projet. Dans les années 1970 est détruit le quartier médiéval Via Madre di Dio. Cette dépréciation se traduit par une forte diminution de la population - de 51809 habitants en 1951 à 21993 en 1991 -, avec un taux élevé de logements inoccupés $(22,1 \%)$.

1. Cet article constitue un premier jalon d'une recherche menée sur les modalités de sécurisation du centre historique de Gênes. Les éléments concernant la patrimonialisation et la gentrification sont issus des terrains menés entre 2003 et 2007, dans le cadre du travail de doctorat. En 2008, une nouvelle mission a porté plus spécifiquement sur les questions de sécurisation et de l'éviction de marginalités, reposant sur des entretiens avec acteurs et habitants et une observation des lieux et pratiques associées. 
Avant le processus de réinvestissement, le centre historique de Gênes connaît des catégorisations sémantiques qui témoignent des valeurs accordées aux espaces et à ses usagers. En effet, le langage participe de la construction et de l'appropriation de l'espace, en permettant ses hiérarchisations (Bulot et Veschambre, 2006). Le centre historique est constitué de multiples ruelles de quelques mètres de large, débouchant sur de petites places, témoignage de l'organisation viaire médiévale. Ces ruelles sont appelés vicoli, ou en génois carruggi. Progressivement s'est opérée une réduction de l'espace urbain à cette trame viaire : aller au centre historique se disait andare nei carruggi, aller dans les ruelles, pour mieux indiquer ce sentiment de désorientation qui saisit le génois extérieur (le bâti devient alors l'élément en creux de cette dénomination, illustration d'un espace que beaucoup ne font alors que traverser, sans poser la question de ses habitants). Ces ruelles sombres apparaissent criminogènes. La presse locale dans les années 1980 et 1990 utilise l'expression nei carruggi pour localiser les crimes dans le centre historique : «nei vicoli della paura », «assassinio nei vicoli ». Le centre historique est réduit aux carruggi, sources de peurs urbaines. La désignation d'un quartier par ses paysages urbains les plus emblématiques pour signifier l'insécurité accroit l'idée d'une forme urbaine criminogène, à laquelle s'ajoute l'insalubrité. Là aussi se joue un essentialisme typomorphologique : l'absence de lumière, l'étroitesse des ruelles, la superposition d'étages de différentes époques, expliqueraient cette insalubrité, et nécessitent donc un traitement urbain de cet espace, d'abord dans le cadre classique des destructions de quartiers ou d'îlots, puis dans des opérations plus ciblées d'une dédensification par la destruction d'édifices, puis la mise en avant des appartements les plus lumineux et aérés, cible de la revalorisation et objets des plus-values immobilières les plus conséquentes.

Certaines rues deviennent emblématiques de différentes formes de marginalité et de marginaux : la mafia via di Pré, la prostitution dans les via della Maddalena et via del Campo, la toxicodépendance dans les vicoli, tandis que le tissu social populaire composite est ramené à ces figures. Ainsi sont construits des espaces répulsifs pour les citadins extérieurs à ce quartier, en même temps que des types sociaux emblématiques. L'arrivée des migrants d'Afrique et d'Amérique Latine à la fin des années 1980, installés dans leur très grande majorité dans le centre historique, au départ sous la coupe de marchands de sommeil, ajoute de nouvelles catégorisations et typifications péjoratives. L'échec du programme de réhabilitation globale du quartier de Pré dans les années 1980 accentue la dégradation urbanistique des espaces, puisque l'expropriation d'une partie des édifices n'est pas suivie de leur transformation, induisant des formes d'occupation informelle.

Le centre historique est construit comme espace à problèmes. À la fin des années 1990 et au début des années 2000, pour justifier les nombreux programmes publics d’intervention, la municipalité qualifie la situation du centre historique de «degrado sociale» (Comune di Genova, 2002), et inclut de façon cumulative et systémique la marginalisation spatiale, le remplacement du « tissu social historiquement implanté » par l'immigration de l'Italie méridionale après la seconde guerre mondiale et récemment des pays du Sud, les dégradations du bâti, la dégradation économique et les enjeux sociaux.

Comment cette déqualification initiale, partagée par un grand nombre d'habitants extérieurs au centre historique, véhiculée chaque jour dans les médias locaux, influençant les mobilités quotidiennes, a-t-elle été dépassée pour permettre une revalorisation et un réinvestissement économique? Que deviennent dans le processus de régénération les usages anciens et leurs discours? Les mots qui semblaient ancrés, rivés au centre historique de Gênes ont-ils été balayés par de nouvelles appropriations des espaces?

\section{DE LA DÉQUALIFICATION À LA PATRIMONIALISATION ET GENTRIFICATION}

Résultat d'un processus (Choay, 1999), le patrimoine n'existe que par le regard qui le constitue, ouvrant la voie à l'étude des controverses sur la patrimonialisation (Gravari-Barbas et Veschambre, 2003). Le patrimoine constitue, en tant qu'il légitime ou disqualifie des usages des espaces, un instrument d'appropriation d'un espace (Veschambre, 2008), préalable à un changement social, 
aboutissant dans les espaces urbains centraux à une gentrification (Semmoud, 2004). Ce processus semble se généraliser à l'ensemble des villes dans le monde (Smith, 2003), constituant une des modalités du réinvestissement immobilier d'espaces délaissés, Gênes constituant un cas particulier de la requalification des friches portuaires et urbaines en Méditerranée (Rodrigues-Malta, 2004), relevant d'un savoir partagé par les acteurs locaux.

Dans ces espaces urbains anciens, la déqualification générale préalable se transforme en déqualification des usages antérieurs désormais incompatibles avec ce qui devient un patrimoine à redécouvrir et révéler. À Gênes, le centre historique est perçu à partir des années 1970 comme une ressource, et plus seulement un problème urbanistique. Les palais et églises aux façades abîmées, d’origine médiévale, ou Renaissance dans la via Garibaldi, sont l'objet d'un réinvestissement patrimonial et économique visant à les détacher de leur usage social actuel. Ainsi la restauration du Palazzo Ducale en 1992 implique une normalisation de ses usages, par éviction des locataires et commerçants qui occupent les lieux, remplacés par des commerces culturels et récréatifs, tandis que les salles sont transformées en espaces d'exposition et de représentation. De même, les anciens entrepôts portuaires sont vidés de leurs occupants avant la réhabilitation et réutilisation, et réaménagement après requalification urbaine, par exemple pour la réalisation du Musée de la Mer inauguré en 2004 pour Gênes capitale européenne de la culture.

Or la déqualification préalable à Gênes constitue une condition de la revalorisation, favorisant leur appropriation et rentabilisation. La déqualification et la description d'un état d'urgence (basé sur la situation du bâti, sur des urgences sanitaires et sociales) permettent de légitimer l'action publique dans cet espace, elle-même destinée à garantir la viabilité économique des investissements privés, à l'imitation du modèle barcelonais, comme l'indique l'adjoint à l'urbanisme entre 1997 et 2006, Bruno Gabrielli (entretien). Un entretien mené avec un des principaux agents immobiliers spécialisés dans le centre historique confirme l'existence d'un rent gap (Smith, 2002) dans l'esprit des investisseurs. Ainsi entre 1998 et 2003 le centre historique connaît la hausse des prix immobiliers la plus importante de Gênes, de $43 \%$, atteignant 1600 euros le m² (Il Secolo XIX, 5 mars 2004), moyenne qui cache en outre des disparités importantes, la gentrification du centre historique étant incomplète ${ }^{2}$, avec une gentrification en mosaïque.

Les acteurs privés se calent sur ces mutations impulsées par les acteurs publics. Les familles génoises appartenant aux élites locales réinvestissent dans leurs demeures du centre historique de façon concomitante aux politiques publiques, par exemple en restaurant les façades des palais des Rolli à partir des années 1990 alors qu’ils en sont propriétaires depuis des décennies voire plusieurs siècles (cas du palais Lomellini, partiellement transformé en musée par ses propriétaires). Il y a ainsi synchronie entre investissements publics et privés, manifestant une requalification pas seulement urbanistique, mais qui opère également par conviction, s'immisçant dans les débats citoyens, les programmes politiques, les projets de ville. Le projet urbanistique a pu être mené car il opérait sur le fond d'un consensus large seul capable de mobiliser capitaux publics et privés (Jacquot, 2007). Les espaces auparavant déqualifiés sont remis en avant sur le registre de la redécouverte, du dévoilement, ce qui permet de considérer les usages antérieurs comme une parenthèse à effacer. Cette thématique de la redécouverte irradie également les discours des gentrifiers, qui mettent en scène leur propre découverte d'un patrimoine délaissé, en recherchant sous les enduits et peintures au plafond les traces de fresques du XVII ${ }^{\text {e }}$ siècle. Ainsi la gentrification, modalité d'appropriation des espaces urbains par le réinvestissement immobilier, se déploie sur le fond d'une déqualification préalable des espaces transformée en déqualification des usages antérieurs.

2. La gentrification est incomplète pour trois raisons. Tout d'abord, quelques axes étaient demeurés habités par la bourgeoisie locale et la requalification des espaces s'est traduite par une simple restauration du bâti, sans qu'il y ait, là, une gentrification. Dans les autres espaces du centre historique, des îlots voire groupes d'îlots demeurent paupérisés, notamment du fait d'un peuplement d'origine étrangère, le centre demeurant un espace de transition pour les primo-arrivants. Enfin, dans certains édifices, seuls les étages supérieurs connaissent un processus de changement social effectif, les premiers étages demeurant très sombres du fait de l'étroitesse des voies et de la hauteur du bâti médiéval. 
Toutefois cette appropriation socialement plus sélective n’est pas stabilisée et nécessite des dispositifs de contrôle et d'éviction des usages et usagers jugés illégitimes. La sécurisation des espaces est un des instruments accompagnant le réinvestissement du patrimoine.

\section{La sécurisation des espaces}

Les diverses formes de marginalité et de petite délinquance sont perçues comme un frein au réinvestissement de cet espace. La normalisation des espaces par la sécurisation et l'éviction d'usages et d'usagers constitue un des instruments de l'intervention urbanistique, concomitante de la réappropriation.

\section{Patrimoine et SÉCurisation des ESPACES}

La sécurisation renvoie aux dispositifs et politiques de production de sécurité. Toutefois la sécurité n'est pas un bien public diffus mais s'adresse à des groupes variables, et peut être interprétée comme un élément renforçant une appropriation socialement sélective. Le " dispositif » désigne la solidification de discours, d'aménagements, d'institutions, agissant dans le cadre d'une stratégie de pouvoir (Beuscart et Peerbaye, 2006; Foucault, 1994, 2001a), ie d'une stratégie orientant les actions des individus (Foucault, 2001b). Dans ce cadre, la sécurisation des espaces peut être analysée comme mise en place d'un dispositif, basé sur des discours sur la ville, un urbanisme de la sécurité (Bourdin, 2004) et des techniques particulières (vidéosurveillance, fermeture, statut des espaces, etc.), des règlements, opérant par différenciation des individus ou des usages basée sur leur légitimité supposée à occuper un espace (Morelle, 2006).

Les politiques de régénération urbaine intègrent de façon croissante des dispositifs de sécurisation des espaces (Bannister et Fyfe, 2001 ; Coleman et Sim, 2000), mettant en avant la nécessité du contrôle de populations et la définition d'un ordre social légitime (Coleman et al., 2005). La sécurisation des espaces se cale également sur les nouvelles modalités de régulation urbaine, basées sur des partenariats publics/privés, notamment via les Business Improvement Districts (Steel et Symes, 2005) ou les agences publiques/privées de régénération urbaine, permettant une « corporatisation of crime control » (Coleman et al., 2005). Or ces dispositifs de sécurisation impliquent également la définition des pratiques désormais jugées illégitimes, accolés à des catégories d'usagers, parfois construits comme «usuals suspects » (Coleman et al., 2005).

\section{GÊNES ET LA PRODUCTION MULTIFORME DE LA SÉCURITÉ}

La sécurisation des espaces est une dimension prégnante de la transformation du centre historique de Gênes. Toutefois elle ne relève pas d'un foyer unique et se trouve au contraire au croisement de différents dispositifs et principes, publics et privés, formels et informels : entre les rondes organisées de nuit par certains habitants et commerçants et les aménagements urbains à partir d'implantations commerciales et d'éclairage se jouent des principes et des registres de légitimation de la sécurisation différents. La sécurité n'est en effet pas un bien neutre et relève d'une production multi-focale : les différentes formes de production de sécurité sont autant de modalités de maîtrise des usages et des usagers de l'espace, en appui, en complément ou en alternative aux opérations de régénération urbaine menées.

La prégnance de la sécurisation dans l'action urbanistique communale a varié : d'abord noyée dans les programmes généraux, elle devient en 2007 un thème indépendant de l'action publique locale. L'ancien maire de Gênes Giuseppe Pericu entre 1997 et 2007, dans son ouvrage-bilan (Pericu et Leiss, 2007), n'aborde pas la question de la sécurité, à la fois en raison du traumatisme lié à l'hypersécurisation du centre historique lors du G8 organisé par le gouvernement italien à Gênes en 2001 et pour des motifs plus principiels : la sécurité est à la fois une conséquence et un instrument du renouvellement urbain (Comune di Genova, 2002), et pas un objet d'intervention en 
soi. Le programme Urban 2 par exemple, dont le périmètre d'intervention coïncide avec le centre historique, pose l'hypothèse que toutes les dimensions de cet espace fonctionnent en interaction et que l'attractivité du lieu repose sur un sentiment de sécurité, tout en contribuant à le renforcer.

Toutefois, l'élection du nouveau maire Marta Vincenzi entraîne une prise en compte accrue de la sécurité, avec la création d'un poste d'adjoint désormais dévolu à la question de la sécurité urbaine (assessore città sicura, F. Scidone). Cela rejoint une réorientation des politiques régionales et provinciales vers la mise en avant de la sécurité urbaine, avec la création d'un observatoire régional sur la sécurité urbaine, dirigé par Stefano Padovano (2006), mais surtout la volonté du gouvernement national d'impliquer les collectivités dans la production de sécurité urbaine. Ainsi sont conclus entre le ministère de l'intérieur, la commune et la préfecture des accords de sécurité (patto di sicurezza, à Gênes, le 14 juin 2007), tandis que le décret du 5 août 2008 définit une "sécurité urbaine », au nom de laquelle le maire dispose de pouvoirs plus étendus, par exemple pour lutter contre la prostitution.

Cette création est également destinée à combler une partie des attentes citadines, qui formulent les problèmes du centre historique plus directement en termes de sécurité (Guarnero et Simonetti, 2007) et cela depuis les prémisses du processus de requalification urbaine, mettant en avant une demande différente de sécurisation. À la fin des années 1980 se créent des comités de quartiers, impliquant plusieurs centaines d'habitants, réclamant de meilleures conditions de vie et des actions publiques conséquentes, se jugeant délaissés par la municipalité. La sécurisation des espaces constitue la revendication principale, puisque sont fustigées la présence des toxicomanes et les seringues qui jonchent le sol, la délinquance et la micro criminalité, alors que la préservation patrimoniale ne constitue pas pour eux une priorité. Ces comités prennent ensuite des voies divergentes : un groupe d'habitants, proche des milieux universitaires et architectes, met en œuvre une méthode participative pour une réhabilitation du quartier Vigne (Jacquot, 2009), tandis que quelques comités se rapprochent de la droite locale et dénoncent de façon accrue la présence des immigrés au sein du centre historique. L'actuel président du conseil de quartier du centre historique relaie ces attentes formulées sur un strict plan sécuritaire.

Ce lien entre sécurité, régénération urbaine et appropriation des espaces apparaît plus nettement dans le balancement entre deux procédés de sécurisation : la fermeture des espaces et la mise en circulation des usagers.

\section{Circuler ou fermer, techniques de SÉCurisation eT de rÉAPPropriation des espaces}

Un des aspects importants de la sécurisation des espaces, illustrant son caractère protéiforme en même temps que le lien avec la réappropriation des espaces, est l'articulation entre circulation et fermeture. Dans un premier temps, la sécurisation des espaces est envisagée comme conséquence du renouvellement urbain. En effet, la délinquance est analysée par les acteurs publics en terme de désappropriation de l'espace public $^{3}$ : favoriser une réappropriation de ces espaces grâce à une circulation accrue permettrait alors de les sécuriser. Cette réappropriation signifie la fin de la maîtrise des espaces par des groupes identifiés comme délictueux. La circulation est donc un principe de sécurisation. Cette idée-force de l'action publique se traduit par plusieurs interventions, destinées à augmenter dans le centre historique la présence de consommateurs, de touristes et d'étudiants, comme l'indique le Piano di Sicurezza établi dans le cadre du programme européen Urban 2.

Ainsi la création de nouveaux bâtiments universitaires sur les friches portuaires du quartier Darsena, à proximité du nouveau musée de la Mer, génère des parcours entre les universités établies dans les anciennes demeures du centre historique (via Balbi) et les nouveaux établissements, traversant la via Pré, voie étroite comportant de nombreuses impasses jugées criminogènes. De même, la création de nouveaux commerces est encouragée via le dispositif de l'incubateur

3. Pour le chercheur il s'agit bien d'une modalité particulière d'appropriation de l'espace. 
d'entreprises (loi régionale Bersani 266/97, partenariat entre la région et la commune), dans les espaces jugés les plus dégradés, dans une optique de reconquête face à certaines marginalités. Des dizaines de commerces sont ainsi ouverts depuis 10 ans : restaurants, librairies, œnothèques, boutiques d'artisanat, etc. Le public-cible de ces commerces est constitué des nouveaux usagers du centre historique. La dernière zone d'intervention de l'incubateur est la place dei Truogoli di Santa Brigida, qui jouxte la via di Pré, objet d'interventions urbaines importantes : restaurations et réhabilitations des édifices, interventions sur l'espace public et les accès, éclairages. Là sont implantés des commerces destinés à du shopping touristique ou de loisirs : librairie de voyage, boutique de randonnée, restaurant. Les commerçants exercent en outre une action de contrôle informel des espaces publics, faisant fuir les personnes jugées indésirables, appelant les forces de l'ordre, contribuant à une réappropriation des lieux.

Les nouveaux usagers du centre historique sont ainsi des adjuvants de la sécurisation des espaces, par la propre déambulation de leur corps, en ne laissant aucun espace disponible pour des activités jugées illicites. La réappropriation suppose le mouvement des corps. Ce principe est également présent parmi certains comités de quartier, ainsi le Comitato Santa Brigida, qui a créé un modèle de parcours touristique, avec l'idée que des passages plus nombreux permettent une désappropriation par des groupes marginaux et une "réappropriation du territoire » (entretien en 2008). L'appropriation passe ainsi par une désappropriation basée sur des flux circulatoires.

Toutefois se développe également une autre pratique, qui peut sembler contradictoire : la fermeture de certaines ruelles, là aussi pour dissuader des occupations jugées illégitimes. Deux cas permettent d'illustrer cela. Pour réhabiliter une dent creuse issue de la seconde guerre mondiale, la commune met en place un processus participatif avec les habitants, pour déterminer le devenir de ce lieu qui devient la piazza dei bambini, place fermée par des grilles le soir. L'établissement de nouvelles règles (horaires d'ouverture, contrôle du voisinage accru) est explicitement une façon d'empêcher certains usages, par exemple les fêtes alcoolisées des migrants masculins sud-américains, et constitue donc une désappropriation de lieux. L'autre cas est davantage diffus, illustré par les nombreuses portes installées dans le centre historique pour fermer passages, ruelles ou impasses précédemment utilisés pour des activités illicites, notamment la consommation de drogues. La municipalité valide a posteriori l'installation des portails, après demande de groupes d'habitants, mais doit gérer ensuite les protestations d'autres habitants. Des questions telles que l'ouverture ou non en journée des portails, la possession des clefs, l'appropriation, provoquent des conflits localisés, entraînant des actes de vandalisme (détérioration des serrures, par exemple salita Famagosta), et des pétitions d'autres habitants et comités opposés à la fermeture de rues, qui y voient une façon de faire augmenter les prix immobiliers, par l'argument d'une sécurisation accrue, et critiquent la privatisation de fait.

L'appropriation présuppose ainsi la mise en place de discours et dispositifs cristallisés, qui se manifestent par la sécurisation des espaces. Au-delà de l'appropriation se joue la désappropriation des espaces, passant notamment par l'éviction de catégories d'usagers.

\section{Refoulement et retour des marginalités, l'exemple de la prostitution}

L'appropriation des espaces au-delà du dyptique fermeture/mouvement passe par une éviction d'usages et d'usagers désormais perçus comme incompatibles, par expropriation, criminalisation, pressions ou incitations. Ainsi peut être mis en évidence un lien entre gentrification et éviction de la prostitution des quartiers historiques (Hubbard, 2004), notamment dans le cadre de la ville entrepreneuriale (Séchet, 2009).

\section{L'ÉVICTION DE LA PROSTITUTION...}

La prostitution à Gênes témoigne de tels enjeux de réappropriation par éviction. En effet, la prostitution devient une cible de l'intervention urbaine et de la normalisation des espaces, notam- 
ment depuis 2007. La prostitution dans le centre historique connaît de multiples localisations, en fonction notamment de l'origine et du sexe des prostitués, et concerne à la fois des ruelles étroites, avec ses bassi, locaux au rez-de-chaussée des édifices, considérés souvent comme insalubres, et certaines rues importantes du centre historique, notamment via della Maddalena. L'éviction ne peut se faire directement, dans la mesure où la prostitution n'est pas illégale en Italie, à l'inverse du proxénétisme qui concerne une grande partie des prostituées nigérianes, de façon plus incertaine les prostituées venues d'Amérique Latine qui entrent dans le cadre d'une prostitution « consensuelle » liée à la nécessité de rembourser un prêt important (Abbatecola, 2005). Plusieurs actions sont alors menées : contrôles plus fréquents, enquête auprès des propriétaires des bassi ${ }^{4}$, mais surtout, action orientée vers l'espace urbain lui-même. Ainsi la maire Marta Vincenzi met en place en août 2008 une ordonnance concernant les bassi, en définissant des usages particuliers à ces locaux, et en imposant pour les bars des vitres transparentes, décourageant ainsi l'activité sexuelle et favorisant des activités jugées plus conformes au centre historique. En effet, le cœur de la prostitution étrangère se trouvait dans les ruelles qui partaient de la via Garibaldi, axe majeur du secteur Patrimoine Mondial de l'Humanité depuis 2006 et transformée en axe muséal. Les contrôles associés à cette ordonnance permettent la fermeture de nombreux bassi associés à la prostitution, témoignant du lien entre éviction de la prostitution, gentrification et réinvestissement des espaces urbains.

\section{...OU ÉVICTION DE PROSTITUÉS?}

Toutefois la prostitution génoise n'est pas perçue comme homogène, ce qui induit des distinctions et catégorisations internes entrainant des stratégies différenciées d'éviction. En effet, les multiples facettes de la prostitution cohabitant dans le centre historique de Gênes ne sont pas perçues de la même façon. Ainsi dans le quartier Ghetto existe une prostitution transsexuelle, avec une activité davantage établie, un aménagement plus conséquent de l'espace public, une densité plus importante dans les ruelles. Le quartier compte une trentaine de transsexuels établi.e.s à leur compte, souvent propriétaires du basso et vivant également dans le quartier. Or leur présence est jugée plus légitime. Ainsi dans le cadre du Contratto di quartiere mis en place par la municipalité, avec des financements nationaux, le processus participatif auprès des habitants inclut les représentants des transsexuels. De façon informelle au sein du service Città sicura est relayée l'opinion selon laquelle ils contribuent par leur présence continue à la sécurisation d'un quartier encore populaire avant l'intervention. Souvent Italiens, implantés depuis longtemps, ils sont considérés comme appartenant au quartier. Au moment de l'ordonnance anti-bassi, les trans reçoivent le soutien de nombreux acteurs sociaux, notamment de la communauté catholique San Benedetto et du charismatique prêtre des marginaux Don Gallo, qui permettent la médiation avec l'assesseur à la sécurité et obtiennent que le Ghetto ne soit pas concerné par la fermeture des bassi.

Ainsi ce n'est pas l'activité mais la catégorisation des actifs qui polarise les motivations à l'éviction, et notamment l'origine étrangère des prostituées selon des membres de l'unità di strada qui gère le projet «Oltra la strada », tandis que la prostituée italienne ou le trans font partie d'un imaginaire local réactivé. À rebours donc des analyses univoques mettant en avant régénération urbaine et éviction des marginalités, comment expliquer ces différenciations?

\section{LE RETOUR DU REFOULÉ : IMAGINAIRES ET MARGINALITÉS}

Ce traitement différencié de la prostitution pose la question des imaginaires sur lesquels reposent la prostitution, et leur rôle dans l'appropriation des lieux. En effet, les marginalités sont parées de signifiés contradictoires, à la fois menaces et marqueurs de modes de vie particuliers au

4. Cette enquête a permis, par exemple, en 2009, l'arrestation d'une famille originaire de Calabre (la maire M. Vincenzi justifiait notamment sa politique par les liens entre prostitution et mafia), qui possédait 78 bassi (La Repubblica, 04/07/2009), destinés à la prostitution et au logement d'immigrés entassés dans des locaux insalubres. 
sein du centre historique, allant jusqu'à une forme de fascination pour l'encanaillement permis dans ces espaces (Dal Lago et Quadrelli, 2006). La nouvelle sociabilité qui se construit dans le centre historique parmi ses nouveaux usagers repose sur des imaginaires de la transgression, qui supposent le maintien de discours ou de traits emblématiques de ce qui dans le même temps est refoulé. La réactivation de la marginalité sur un mode idéel est mise au service de l'appropriation elle-même, niant l'idée d'une appropriation socialement exclusive.

Ainsi, les récits des nouveaux habitants dessinent un rapport enchanté aux lieux et à ses habitants, certains devenant des archétypes imaginés de rapports sociaux transgressifs. Ces gentrifiers qui se présentent comme pionniers évoquent souvent la prostituée de leur immeuble ou quartier, généralement italienne, plutôt âgée ${ }^{5}$, témoin d'un autre temps du centre historique. Dans ces récits, elle est la voisine quelques étages plus bas, qui vous salue toujours, elle est la cliente de la boulangerie que vous fréquentez, elle apprend le prénom de vos enfants, bref la prostituée est un personnage social du centre historique, isolé, décontextualisé. Cette prostituée âgée est également celle d'avant les migrations et dessine en creux une sociabilité rêvée, faite de personnages quasi publics haut en couleurs, dessinant un âge d'or populaire du quartier. De même, les visites organisées à partir des années 1990 par l'association Genova insieme pour les habitants de Gênes d'autres quartiers souhaitant redécouvrir le centre historique mettent en scène de telles rencontres avec la prostituée emblématique, qui les interpelle, pose des questions, et devient un des marqueurs de cette redécouverte. Ces imaginaires décontextualisés (déconnectés des conditions d'exercice et des mutations de la prostitution génoise) mais recontextualisants (au sens où ils participent de la construction d'un nouveau rapport aux lieux) reposent sur l'idée d'une prostitution à Gênes consubstantielle au centre historique et à ses sociabilités. Le chanteur populaire génois Fabrizio de André évoque dans de nombreux textes (par exemple «Boca di Rosa ») les prostitués et transsexuels de la via del Campo et du centre historique dans les années 1970, tandis que divers ouvrages sur la ville mettent en avant la présence ancienne de la prostitution, dès le Moyen Âge (Borzini, 2004). La littérature policière locale (gialli) qui se développe dans les années 2000 mobilise souvent le personnage de la prostituée, victime, témoin, compagne de l'enquêteur, par exemple dans les romans policiers de P. Pettinotti, E. Ratto ou B. Morchio. Ces différents textes construisent la légitimité de la présence des prostituées dans le centre historique par-delà les tentatives d'éviction.

En janvier 2009 un accord informel est pris entre la municipalité et les prostitués du centre historique librement établis, qui s'engagent à des comportements et vêtements plus discrets (à une visibilité moindre donc), à l'entretien des ruelles, au respect du voisinage, mais doivent signaler les cas de proxénétisme et de microcriminalité. La légitimité de la présence d'une forme de prostitution, ainsi qu'un effet de régularisation territoriale, sont ainsi, quelques mois après les mesures anti-bassi, reconnus. Ce pré-accord résulte de la mobilisation des prostitués et d'acteurs sociaux à partir de l'été 2008, de la reconnaissance par la municipalité d'une forme d'impuissance à évincer la prostitution du centre historique, mais a également été facilité à Gênes par cet imaginaire de la place de la prostituée dans la société locale, alors que bien d'autres villes italiennes, telles Pise ou Rome, durcissent la répression de la prostitution.

La catégorisation des espaces et usagers dans le cadre des stratégies et modalités de réappropriation se trouve ainsi dédoublée. L'éviction des marginalités participe bien des stratégies de revalorisation des espaces, mais ces marginalités deviennent sur un plan idéel des éléments de valorisation, mettant en scène une sociabilité typifiée et apaisée. Se joue ainsi une forme de réflexivité de la marginalité. Les catégorisations deviennent alors plus fines, distinguant diverses formes de prostitution et catégories de prostituées, et permettant même le maintien de ce qui en première analyse semble refoulé. 


\section{Conclusion}

Le centre historique de Gênes connaît un processus concomitant de patrimonialisation et de gentrification, sur fond d'une déqualification générale des espaces qui laisse place à une déqualification des usages et usagers antérieurs. Toutefois ces processus ne sont pas achevés, et nécessitent la mise en place de discours et dispositifs supports de cette réappropriation des espaces, pour la pérenniser dans un contrôle constant des pratiques.

Plus qu'un usage exclusif, il s'agit de mettre en œuvre un contrôle des espaces. Les techniques de sécurisation montrent le caractère protéiforme et multifocale de cette sécurisation, à travers l'usage paradoxal de l'ouverture et de la fermeture des espaces. L’ouverture est théorisée par les pouvoirs publics, par la mise en mouvement des espaces par la circulation et les flux d'usagers, habitants ou touristes, contestant partout par leurs corps en déplacement les appropriations jugées illégitimes, tandis que les fermetures constituent les réponses à des demandes privées de sécurité et sanctionnent des échecs temporaires en même temps que des stratégies de privatisation qui se greffent sur cette volonté de normalisation généralisée des usages au sein du centre historique. L'éviction d'usagers jugés illégitimes, notamment la prostitution, illustre également cette volonté de contrôle comme condition d'une appropriation. Toutefois ces usages antérieurs ne disparaissent pas et refont surface sous la forme d’une sociabilité idéalisée, qui neutralise sur un plan idéel la dimension sociale de l'appropriation des espaces. Se jouent ainsi dans ces stratégies d'appropriation, par déqualification, contrôle, éviction, la définition des usages et usagers légitimes des espaces, mais également la pérennisation de l'appropriation.

\section{Bibliographie}

Авbatecola E., 2005. Donne al margine, la prostituzione straniera a Genova, Fratelli Frilli Editore, 204 p. Bannister N.-R., Fyfe J., 2001. Introduction, fear and the city, Urban studies, vol. 38, n 5-6, p. 807-813. Beuscart J.-S., Peerbaye A., 2006. Histoires de dispositifs, Terrains E travaux, vol. 2, n 11, p. 3-15. Borzini R., 2004. Il malamore, Fratelli Frilli Editori, 182 p.

Bourdin A., 2004. Un urbanisme de la sécurité, dans Bourdin A., Un urbanisme des modes de vie, Moniteur, Paris, p. 35-50.

Bulot T., Veschambre V. (dir.), 2006. Mots, traces et marques. Dimensions spatiale et linguistique de la mémoire urbaine, L'Harmattan, Paris, 246 p.

Choay F., 1999. L'allégorie du patrimoine, Seuil, Paris, 270 p.

Coleman R., Sim J., 2000. You'll never walk alone, CCTV surveillance, order, and neoliberal rule in Liverpool city centre, British Journal of Sociology, vol. 51, n 4, p. 623-639.

Coleman R., Tombs S., Whyte D., 2005. Capital, crime control and statecraft in the entrepreneurial city, Urban studies, vol. 14, n 13, p. 2511-2530.

Comune di Genova, 2002. Il piano operativo per il centro storico, $321 \mathrm{p}$.

Dal Lago A, Quadrelli E., 2006. La città e le ombre, Crimini, criminali, cittadini, Feltrinelli, Milan, 402 p.

Foucault M., 1994. Histoire de la sexualité 1, La volonté de savoir, Paris, Gallimard Tel, 248 p.

Foucault M., 2001b (1977). Le jeu de Michel Foucault, Dits et écrits, Tome II 1976-1988, Paris, Gallimard Quarto, p. 298-329.

Foucault M., 2001b (1982). Le sujet et le pouvoir, Dits et écrits, Tome II 1976-1988, Paris, Gallimard Quarto, p. 1041-1062.

Gravari-Barbas M., Veschambre V., 2003. Patrimoine : derrière l'idée de consensus, les enjeux d'appropriation de l'espace et des conflits, dans Melé P., Larrue C., Rosemberg M. (coord.), Conflits et territoires, Tours, PUFR, MSH Villes et territoires, p. 67-82.

Greffe X., 2003. La valorisation économique du patrimoine, La documentation française, Paris, 384 p.

Guarnero F., Simonetti C., 2007. Genova profunda, ombre e luci del più grande centro storico d'Europa, due carruggiai lo raccontano con amarezza e ironia, Erga edizioni, 116 p. 
Hubbard P., 2004. Cleansing the metropolis: sex work and the politics of zero tolerance, Urban Studies, vol. 41, nº 9, p. 1687-1702.

JaCQUOT S., 2007. Le réinvestissement public et privé des espaces historiques centraux, une étude comparée de Gênes, Valparaiso et Liverpool, Thèse de doctorat en géographie, Université d'Angers, 664 p.

JACQUOT S., 2009. La participation au service de la régulation urbaine dans le cadre des projets urbains et patrimoniaux à Gênes et Valparaiso, Geographica Helvetica, vol. 4, p. 227-235.

Morelle M., 2006. Les enfants des rues, l'État et les ONG, qui produit l'espace urbain? Les exemples de Yaoundé et d'Antananarivo, Afrique contemporaine, n² 217, p. 217-229.

Padovano S., 2005. La paura e il crimine, primo rapporto sulla sicurezza urbana in Liguria, Carocci pressonline, regione Liguria, 143 p.

Pericu G., Leiss A., 2007. Genova nuova, la città e il mutamento, con un dialogo tra Giuseppe Pericu e Renzo Piano, Roma, Interventi Donzelli editore, $141 \mathrm{p}$.

Redfenn P.-A., 2003. What makes gentrification?, Urban Studies, Vol. 40, nº 12, p. 2351-2366.

Riegl A., 1984. Le culte moderne des monuments, Son essence et sa genèse, Paris, Le Seuil, 122 p.

Ripoll F., Veschambre V., Introduction : l'appropriation de l'espace comme problématique, Norois, n 195 , p. 7-15.

Rodrigues-Malta R., 2004. Une vitrine métropolitaine sur les quais, villes portuaires du Sud de l'Europe, Les Annales de la Recherche Urbaine, n 97, p. 93-101.

SÉchet R., 2009, La prostitution, enjeu de géographie morale dans la ville entrepreneuriale. Lectures par les géographes anglophones, L'espace géographique, vol. 38, p. 59-72.

Semmoud N., 2004. Valorisation patrimoniale et changement social : un pléonasme?, dans Gravari-barbas M., Habiter le patrimoine, enjeux - approches - vécu, Rennes, PUR, p. 265-280.

Sмiтн N., 2002 (1996). The new urban frontier, Gentrification and the revanchist city, London/New York, Routledge, $262 \mathrm{p}$.

Sмiтh N., 2003, La gentrification généralisée, d'une anomalie locale à la régénération urbaine comme stratégie urbaine globale, dans Bidou-Zachariasen C. (dir.), Retours en ville, Descartes \& Cie, Paris, p. 45-72.

Steel M., Symes M., 2005. The privatisation of Public Space?, The American Experience of Business Improvement Districts and their relationship, Local Government Studies, Vol. 31, n 3, p. 321-334.

Tomas F., 2004, Les temporalités du patrimoine et de l'aménagement urbain, Géocarrefour, vol. 79, n³ 3 , p. 197-212.

Veschambre V., 2008. Traces et mémoires urbaines, enjeux sociaux de la patrimonialisation et de la destruction, Rennes, PUR, 315 p.

Vivant E., Charmes E, 2008. La gentrification et ses pionniers, le rôle des artistes off en question, Métropoles, 2008/3, [http://metropoles.revues.org/1972].

Zukin S., 1993. Landscapes of Power, From Detroit to Disneyworld, Los Angeles, University of California Press, 326 p.

Cet article a été reçu le 5 mai 2010 et définitivement accepté le 17 décembre 2010. 